\title{
Disciplinarite et construction interdisciplinaire du savoir sur l'environnement
}

\section{Disciplinaridade e construção interdiplinar do saber ambiental Disciplinarite Disciplinarity and interdisciplinarity construction of the knowledge about environment}

\author{
Edith DELEAGE*
}

\begin{abstract}
RÉSUMÉ
Disciplinarité, intedisciplinaridade et environnement ici sont traités à partir d'une approche hérité de la philosophie occidentale. Le texte se trouve organisé à partir de questions concernant aux changements de civilisation, aux attitudes à deprendre en avant de tels changements, à la nécessité de l'approche interdisciplinaire dans la construction du savoir environnemental, l'urgence de la crise environnementale dans le present, etc. L'analyse de la Modernité se trouve présente au long de toute la réflexion élaborée. Mots-clefs: disciplinarité, interdisciplinarité, environnement, modernité.
\end{abstract}

\section{RESUMO}

A disciplinaridade, intedisciplinaridade e meio ambiente são aqui tratados a partir de uma abordagem herdada da filosofia ocidental. O texto encontra-se organizado a partir de questões relativas à mudanças de civilização, à atitudes a tomar diante de tais mudanças, à necessidade da abordagem interdisciplinar na construção do saber ambiental, à emergência da crise ambiental no presente, etc. A analise da Modernidade encontra-se presente ao longo de toda a reflexão elaborada.

Palavras-chave: disciplinaridade, interdisciplinaridade, meio ambiente, modernidade.

\begin{abstract}
The disciplinaridade, intedisciplinaridade and environment here are dealt with from an inherited boarding the occidental philosophy. The text was organized from relative questions to the changes of civilization, the attitudes to take ahead by such changes, to the necessity of the boarding to interdisciplinar in the construction of environment knowing, to the emergency of the contemporary environmental crisis, etc. The analyzes of the Modernity is showm in all the text.

Key-words: disciplinaridade, interdisciplinaridade, environment, modernity.
\end{abstract}

\footnotetext{
* Antropóloga e filósofa. Professora aposentada do Instituto Universitário de Formação de Mestres/Paris. Professora convidada do Doutorado em Meio Ambiente e Desenvolvimento da UFPR.
} 
Je ne pratique pas une discipline scientifique. Je ne suis ni enseignante, ni chercheure en sciences. Je ne suis pas épistémologue: je n'étudie ni l'histoire, ni les paradigmes, ni les conditions de possibilité d'une science.

Ma formation et ma pratique sont celles du philosopher. C'est pourquoi je traiterai la question "Disciplinarité et construction interdisciplinaire du savoir sur l'environnement" avec les outils conceptuels de la démarche philosophique, ou plutôt d'une démarche héritée de la tradition philosophique occidentale.

"Philosophie occidentale", pour reprendre le terme dont usent le mathématicien Bertrand Russell et l'historien critique des mentalités Geoffrey Lloyd, et dans l'esprit des recherches du philosophe Husserl sur "la crise de la philosophie et les sciences européennes"

I. Ma première piste de réflexion est la suivante : "Serions-nous en train de changer de civilisation?" Et si nous sommes dans cette situation, quelle attitude intellectuelle pouvons-nous et devons-nous avoir pour construire un savoir scientifique?

En effet, la canicule qui a balayé l'Europe cet été 2003 - et qui fit du mois d'août, en France, l'été le plus meurtrier depuis les années 1950 - est apparue aux yeux de tous comme un fait majeur de civilisation.

Incendies de forêt, vagues de chaleur, pollution généralisée par l'ozone, sécheresse dramatique pour des dizaines de milliers de paysans, assèchement des fleuves et des nappes phréatiques sont autant d'épisodes qui mettent en évidence l'extrême fragilité de nos sociétés ivres de leur puissance technologique.

Il y a désormais un ensemble de signes qui confirme l'intuition écologique d'une modification radicale des conditions de vie sur terre. Modification radicale et inédite. Pour la première fois dans l'histoire de l'humanité, celle-ci est capable d'actions dont les effets dangereux sont de dimension cosmique (effet de serre, atteinte à la couche d'ozone, pollution de l'eau, pluies acides, déforestation tropicale, stockage des déchets nucléaires, menace de disparition de maintes espèces vivantes). Et aussi par la maîtrise scientifique du système nerveux, par la maîtrise savante des mécanismes de reproduction, par la maîtrise intellectuelle de l'hérédité, les hommes sont capables d'actions sur les espèces vivantes, de longue portée temporelle. Comme l'écrit le marin et philosophe Michel Serres dans Le contrat naturel, l'inédit c'est que les actions humaines locales ont un effet global.
D'autre part, l'humanité se trouve aujourd'hui agressée par les effets mortifères d'un développement qui était naguère présenté comme le chemin d'un avenir radieux pour tous les habitants de notre planète alors que le modèle dominant de développement n'est pas régi par le principe d'équité sociale, a fortiori pas par celui d'égalité, comme l'ont souligné et le président de l'université et le secrétaire d'Etat à l'Environnement.

Enfin, "L'accumulation met fin à l'impression de hasard". Lorsqu'en 1914 Freud écrit cette phrase à propos de notre rapport à la mort, il ne croit pas si bien dire, puisque la fin du second millénaire fait voir l'accumulation d'excès en tous genres, avec deux guerres mondiales, quarante années d'équilibre de la terreur et la multiplication sans précédent d'accidents majeurs.

Mais le mot de Freud est finalement celui du hasard.

Or, pas plus que la mort, la guerre ou l'accident ne sont des "hasards". Ce sont des résultats indirects de plusieurs facteurs où la démesure l'emporte sur la mesure. Et la démesure ici est celle de la raison instrumentale, c'està-dire celle de la rationalité techno-scientifique (selon le terme du philosophe Gilbert Hottois) qui domine notre civilisation contemporaine.

Ce pourquoi l'architecte et philosophe Paul Virilio écrit: "le vingtième siècle, qui est celui de l'émancipation de la gravité terrestre et de l'acquisition de la vitesse, a également favorisé la croissance exponentielle des catastrophes majeures avec Bhopal, Auschwitz, Tchernobyl", le 11 septembre...

Accident local hier, avec le Titanic ou Seveso, accident global aujourd'hui avec Tchernobyl et la menace terroriste des armes de destruction massive. Ce n'est plus de l'ordre du hasard, mais de celui d'une réalité croissante à l'échelle de cette mondialisation accélérée où attentats et accidents tendent à se confondre dans l'anonymat d'une guerre non déclarée. Alors la question : Comment vivronsnous, et pourrons-nous vivre demain sur terre, n'est pas une question de science fiction.

A. Et les réponses pratiques à cette double question passent d'abord par l'intelligence théorique des innovations scientifiques, des inventions techniques et des accidents qui les accompagnent, dans la mesure même où leur répétition en série devient un phénomène historique clairement repérable.

Pour le naturaliste et philosophe, Aristote, et pour nous hier, l'accident, local, révèle la substance, alors qu'aujourd'hui ce qui arrive, l'accident, global, découvre 
le sens (la signification et la direction) du savoir ou du techno-savoir qui l'a produit.

Dès lors, une recherche scientifique aujourd'hui doit être orientée par un double idéal : celui de savoir pour savoir (recherche fondamentale), et celui de savoir pour prévoir les catastrophes probables des connaissances technoscientifiques contemporaines. Ce que Jean-Pierre Dupuy nomme "le catastrophisme éclairé", et qui est à l'encontre de l'idéal positiviste (comtien) de "savoir pour prévoir et prévoir pour agir".

Cette double idée régulatrice pour toute recherche scientifique implique une attitude intellectuelle non disciplinaire. Pour construire un objet de savoir, dorénavant il faut adopter une attitude intellectuelle anticipatrice de l'accident, à venir, et une attitude intellectuelle éthique responsable et du présent et du futur.

Ce pourquoi le "principe responsabilité" proposé par le gnoséologue et philosophe Hans Jonas pour orienter les recherches et les constructions contemporaines de savoir implique aussi une intelligence nouvelle, celle de la production probable des accidents liée aux techno-sciences.

Prendre en compte cette situation (notre culture techno-scientifique) c'est souligner la nécessité d'un nouvel esprit scientifique, celui de l'interdisciplinarité (sciences physiques et éthique, sciences sociales et éthique).

B. La compréhension de la civilisation actuelle implique d'autre part une "intelligence de la crise de l'intelligence" (la "crise de l'esprit” disait Paul Valéry).

En 1935, dans une conférence prononcée à Vienne, et qui annonçait, hélas, la catastrophe à venir de la barbarie de la Shoah, Husserl, mathématicien et philosophe, dénonçait dans l'attitude scientifique dominante du début $\mathrm{du} \mathrm{XX}^{\mathrm{ème}}$ siècle des lacunes, des aveuglements et des abus.

Des abus liés au scientisme - cette ambition d'une science d'avoir une portée fondatrice et d'être explication ultime à la manière d'une métaphysique.

Des aveuglements liés à l'objectivisme - la raison scientifique oublie, se coupe du sujet, de la sensibilité, de l'esprit, de la conscience, du monde de la vie dans lesquels le savoir s'enracine puisque ce sont des hommes, sujets pensant, sentant et voulant qui constituent le savoir. Coupée de la raison réflexive qui est aussi sensibilité et subjectivité, la raison scientifique devient aveugle.

Des lacunes liées à l'impérialisme scientiste qui conduit à vouloir objectiver et quantifier le sujet lui-même. Une telle approche "objectiviste" et "naturaliste" des vivants humains vide de sens le sujet et la connaissance humaine.
La conséquence de ce fourvoiement de l'attitude qui domine la science est une crise au sens grec du terme crisis, la maladie du sens, due au fait que l'attitude scientifique dominante s'est de plus en plus coupée du sujet (la conscience sensible et réfléchissante), source de tout sens. La science paraît ainsi séparée de la vie, de l'existence. C'est là l'échec contemporain du rationalisme.

Donc la construction d'un savoir aujourd'hui doit veiller à retenir l'enseignement de Husserl: ré-enraciner les sciences dans le monde de la vie. Par là, la construction d'un objet de savoir implique une attitude intellectuelle ambivalente (expliquer et comprendre), attitude qui réfère à deux disciplines scientifiques différentes : sciences de la nature et sciences de l'homme.

II. Ma deuxième piste de réflexion est une deuxième question: pour quelles raisons une attitude intellectuelle interdisciplinaire est-elle nécessaire pour construire du savoir sur l'environnement?

A. Si je cherche - comme le proposait le généalogiste et philosophe Nietzsche - les origines étymologiques et les usages du terme "environnement" parce qu'une langue c'est une culture - pour expliquer de quelle façon cette notion découpe le réel, par cette généalogie du terme "environnement", j'ai une première réponse à ma question.

Environnement est en général considéré comme un terme d'origine anglo-saxonne. Cependant le terme existait en ancien français et il a disparu de la langue au XVI ${ }^{\mathrm{eme}}$ siècle. Il possédait un sens très précis et restreint de : trajectoire circulaire ("le tournoyement et environnement perpétuel du soleil"). Il ne réapparaît en français qu'à partir de 1921, repris de l'anglais environment par Vidal de la Blache dans ses Principes de géographie humaine. L'usage en reste cependant très limité jusqu'au début des années 1960 (on le trouve chez Canguilhem en 1946, ou dans la traduction par Ricoeur des Ideen de Husserl, publiée en 1950). Son introduction dans le Petit Larousse en 1963 corrobore l'amorce d'une diffusion plus importante. Importé de l'anglais, environnement s'est juxtaposé, puis largement substitué, dans un univers en pleine transformation, aux termes utilisés jusque-là pour désigner les divers aspects du monde naturel ou humain auquel il renvoie, comme milieu, nature, paysage, intégrant l'impact du monde industriel, pollutions et déchets. Beaucoup plus qu'à l'effet de l'impérialisme de l'anglais sur notre langue, le succès du terme en français tient au fait qu'il vient combler un manque en introduisant une perspective sans 
équivalent jusque-là dans la culture française, quand l'allemand a su forger Unwelt et l'italien ambiente.

Dès l'origine, environ et environing ont le sens d'encercler ou d'entourer de tous côtés. Environing c'est aussi le fait de baigner dans un fluide comme l'atmosphère ou la lumière, suggérant l'idée d'immersion. Ce terme signifie donc un rapport global, indéfini et non pas limité et étroitement circonscrit, comme le propose environnement en ancien français. Au XIX ${ }^{\text {ème }}$ siècle, quand l'usage commence à s'en répandre, environment possède une double signification : d'une part spatiale, d'encerclement, d'autre part au sens de jeu de relations et d'influences. Environment renvoie donc autant à un monde objectif qu'à un jeu de relations, et donc implicitement à un contexte. Ce n'est véritablement que dans le dernier quart du XIX ${ }^{\text {ème }}$ siècle que le terme environment pénètre le discours scientifique (on ne le trouve pas chez Darwin), en biologie, en géographie et en psychologie avec ce sens d" "entrelacs" selon le terme de Merleau Ponty, philosophe et psychologue.

L'idée contemporaine d'environnement émerge au carrefour de la psychologie (dès 1935 Koffka propose la distinction entre environnement géographique et environnement comportemental et Lewin développe sa théorie du champ), de l'écologie, en particulier dans la version inspirée de la cybernétique, développée par E. P. Odum (1953) et de la prise de conscience de l'impact croissant des activités humaines sur le monde physique dans le contexte du développement économique et industriel qui suit la Seconde Guerre mondiale avec, entre autres, le géographe Sauer, et du développement de la recherche urbaine avec Lynch. Dans les années 70, avec Putnam, le questionnement environnemental joue un rôle central dans l'analyse de la signification. Plus récemment, les neurosciences, prolongeant certains aspects de la phénoménologie, donnent une assise précise à l'idée d'environnement comme élaboration praxéo-cognitive d'un individu en relation. A quoi fait aujourd'hui écho la sociologie d'A. Giddens: environnement est un opérateur de réflexivité pour un individu-acteur fondamentalement en relation. Cette notion ne vaut évidemment pas seulement pour l'homme, mais bien sûr pour l'ensemble du vivant, et permet de tisser des liens entre sciences sociales, éthologie et biologie.

Ainsi, les usages du terme "environnement" impliquent une habitude de penser interdisciplinaire.

B. L'étude des réalités environnementales (des faits découpés par ce terme) nécessite aussi l'interdisciplinarité.
Si la recherche de savoir sur l'environnement a pour objet "des objets hybrides" (selon le terme de Bruno Latour), alors ce savoir élabore inévitablement des concepts globaux qui prennent en compte la complexité de leur réalité et de leur processus d'émergence et d'évolution. Ce sont en particulier la théorie darwinienne de l'évolution des espèces vivantes et l'écologie scientifique fondée par Haeckel qui invitent à supprimer la ligne de démarcation qui, jusquelà, séparait les sciences naturelles des sciences humaines et sociales.

Je m'appuie sur l'ouvrage : L'histoire de l'écologie, une science de l'Homme et de la Nature de Jean-Paul Deléage, physicien et historien de la science écologique, qui montre que cette science inclut l'humain dans les flux du vivant sur notre planète, et que la question de la place de l'homme dans les écosystèmes et plus généralement dans la biosphère, est récurrente dans l'histoire de l'écologie. Elle est posée par le fondateur de l'écologie globale, Wladimir Vernadsky, qui, dès 1926, définit l'homme civilisé des sociétés industrielles comme "une force géologique planétaire". Elle est posée par l'inventeur du concept d'écosystème, Arthur Tansley, qui souligne, dans son article fondateur de 1935, que les activités humaines constituent un facteur biotique décisif d'évolution des écosystèmes.

Ainsi, l'écologie scientifique implique la recherche de concepts capables d'intégrer un ensemble de processus extrêmement vastes, incluant les interventions humaines sur les écosystèmes, sans mettre hors jeu les sciences sociales.

Donc les recherches de savoir sur l'environnement sont inévitablement interdisciplinaires et cela vient de ce que ces savoirs ne relèvent pas du paradigme dualiste moderne. Ce paradigme - que Descartes, physicien et philosophe, conceptualise dans son Traité du Monde, en 1633 - et qui distingue substance pensante et substance étendue, et qui induit de rompre les liens de solidarité entre l'homme et la nature. Ces savoirs relèvent du paradigme ancien, moniste, à repenser. Hajime Nakamura, philosophe et directeur de l'Institut oriental de Tokyo, nous enseigne que ce paradigme dominant la philosophie indienne et le bouddhisme, est à réactualiser : "Auparavant nature (shizen) signifiait l'environnement dans sa globalité, dont l'homme est une partie. La nature étant pensée comme un principe de vie : ce qui va de soi dans les êtres et les choses, un donné que l'on accepte pour ce qu'il est et que l'homme n'est pas fondé à récuser... Nous devons repenser le sens de notre présence au monde, redécouvrir la relation unitaire de l'homme à la nature." 
DELEAGE, E. Disciplinarite et construction interdisciplinaire du savoir...

Et aussi ces savoirs induisent une conception non cartésienne du temps qui souligne que les effets rejaillissent sur les causes et que seule la logique de l'aléatoire et de l'incertain permet de penser les systèmes ouverts et complexes de l'environnement. "Pour le physicien, souligne Michel Serres, le bruit de fond est la règle, et rare la musique. L'ordre apparaît exceptionnel. C'est le chaos qui est la règle."

Enfin, ces savoirs sur l'environnement impliquent que seule une démarche dialectique, celle des liens et des limites, permet de penser la phusis, ce qui naît et advient, et que l'homme cultive. Ce que Merleau-Ponty nomme "entrelacs", “chiasme", car il s'agit de l'entredeux de la nature et de l'artifice, ce champ de transformation réciproque de l'humain par le naturel et du naturel par l'humain.

Mais ce mode dialectique de penser l'environnement est difficile. Parce que les savoirs interdisciplinaires de l'environnement (sciences naturelles, sciences sociales et écologie font encourir le risque qu'une discipline impose à l'autre ses concepts et ses méthodes. Il y a risque de naturalisme et d'objectivisme : que les comportements sociaux soient naturalisés et objectivés. Et aussi, que les rythmes physiques et biologiques soient conçus sur le modèle des rythmes psychologiques ou sur celui des échanges commerciaux : risque d'anthropomorphisme et d'économisme.

III. Enfin, je conclurai par un troisième élément de réflexion.

Les savoirs de l'environnement mettent en avant "la crise environnementale", peut-être parce qu'il y a erreur d'appréciation et angoisse abusive, comme le disent ceux qui se veulent toujours et encore modernes ... Mais c'est peut-être aussi le symptôme que notre épistémè est traversée par ce que le socio-anthropologue Serge Moscovici appelle "la question naturelle".

Le XVIII ème siècle, en Europe, fut marqué par "la question politique": quel est le meilleur gouvernement? Le XIX ${ }^{\text {ème }}$ siècle a pose la "question sociale" : quelle organisation sociale permet de réduire, voire d'annuler les inégalités parmi les hommes? Le $\mathrm{XX}^{\text {ème }}$ siècle pose la "question naturelle", celle de l'histoire et des relations des hommes dans la nature.

$\mathrm{Ce}$, parce que notre épistémè est celle du "désenchantement du monde", de "l'effacement de l'homme", de la "mort de Dieu", de la fin des grands récits et du principe Espérance ; celle aussi d'une civilisation unidimensionnelle et uniformisante, et de l'impérialisme des technosciences et de leurs accidents.
En effet, nous sommes contraints par les risques induits par l'industrialisation massive et par l'intensification de l'agriculture, comme l'analyse l'agronome et sociologue Estelle Deléage dans le Dictionnaire des risques, sous la direction d'Yves Dupont, et dans son ouvrage récent, Paysans de la parcelle à la planète, socio-anthropologie du réseau agriculture durable. Et le productivisme de ces actions humaines et les prélèvements irréfléchis et irréversibles sur les stocks fossiles, entrent en synergie avec le changement de portée des manipulations du vivant promises par la révolution biologique en cours.

"Nous vivons le contraste entre l'époque encore récente où l'homme vivait à l'abri de la nature et notre époque où la nature se trouve désormais remise à la garde de l'homme."

Si l'on retient cette proposition du philosophe Paul Ricoeur et si elle est confirmée par les recherches scientifiques sur l'environnement, alors la séparation établie par le sociologue Max Weber entre le savant et le politique, pertinente en soi, n'est pas suffisante aujourd'hui pour penser les liens et les limites entre savoirs de l'environnement et décisions politiques. En France, le mouvement social actuel des chercheurs en est, à mon sens, une preuve manifeste.

L'insurrection de dizaines de milliers de chercheurs qui déferle sur la France en ce printemps 2004 est l'emblème fort du rôle de la connaissance dans le capitalisme globalisé à l'aube du XXI ème siècle, centré sur la valorisation du capital immatériel, lui-même fondé sur la connaissance et l'intelligence. Ce capitalisme cognitif constitue, comme l'a écrit André Gorz, le mode sur lequel le capitalisme se constitue lorsque la principale force productive devient un "ensemble de savoirs humains abondants, inépuisables, dont l'usage et le partage accroissent l'étendue et la disponibilité."

Cette insurrection d'une génération doit être comprise comme le redéploiement de la lutte de classe sur un nouveau terrain, celui du contrôle du bien collectif que constituent l'ensemble des savoirs. Ces jeunes chercheurs passent à la réflexion commune et à l'action directe pour se réapproprier et mutualiser leur réflexion et ainsi, comme l'a écrit Naomi Klein, "ils font toucher du doigt la manière dont les problèmes sociaux, écologiques et économiques sont interconnectés."

Ainsi, la discussion sur le problème de la science dans son rapport au pouvoir fait partie aussi de l'interrogation sur la nature interdisciplinaire du savoir de l'environnement. 
DELEAGE, E. Disciplinarite et construction interdisciplinaire du savoir...

Pour conclure, comme me l'a enseigné Hannah Arendt, philosophe et femme engagée, "nous sommes toujours les obligés de ce monde". Et notre monde est ce monde fragile et périssable qui est notre milieu commun de vie. C'est pour cela que dans ce débat sur disciplinarité et interdisciplinarité dans la construction du savoir sur l'environnement dans nos sociétés contemporaines dans lesquelles le partage du savoir et le partage du pouvoir ne sont pas réalisés, il est pertinent de réfléchir à l'articulation entre sciences de l'environnement et engagement politique. 